\title{
Rendimiento académico y rehabilitación de las internas estudiantes del Establecimiento Penitenciario de Mujeres de Cusco, 2016
}

\author{
Flor Ivette Aguirre S., ${ }^{1, a}$, Leoncio Zárate G. ${ }^{\text {2,b }}$ \\ 1. Establecimiento penitenciario de mujeres de Cusco - INPE \\ 2. Universidad Andina del Cusco \\ a Docente de Educación Secundaria; b Físico Matemático
}

\section{Resumen}

Los centros de reclusión penitenciarios en el Perú, han sido creados con la finalidad de rehabilitar a las personas en un régimen cerrado y privados de algunas de sus libertades a través de diferentes procesos, como por ejemplo: Asistencia psicológica; identificación de aspectos intrínsecos de los internos, que hacen que estos sean idóneos para realizar una determinada tarea dentro de la sociedad; inicio o culminación del proceso educativo —en el nivel de primaria y secundaria-. Siendo este último proceso, establecido como una variable de estudio en el presente trabajo de investigación, con el propósito de identificar si tal variable contribuye en el proceso de rehabilitación de las internas en el penal de mujeres de Cusco, durante el periodo 2016. Así, direccionado hacia el objetivo formulado se identificaron los niveles de rendimiento y rehabilitación en los que se encuentran actualmente en dicho penal, seguido de la utilización de pruebas de hipótesis con la finalidad de identificar la relación de rendimiento hacia la rehabilitación a través de un diseño descriptivo correlacional-causal de corte transversal establecido. Del análisis de los datos extraídos, se pudo concluir que la rehabilitación no es afectada significativamente por el rendimiento académico de las internas estudiantes dentro del penal, evidenciándose, en este caso, que la rehabilitación no depende de la variable 'rendimiento académico.'

Palabras clave: Rendimiento académico, rehabilitación, tratamiento penitenciario, establecimiento penitenciario. 


\begin{abstract}
Academic performance and rehabilitation of internal students of the penitentiary establishment of women in Cusco, 2016

Penitentiary detention centers in Peru have been created with the aim of rehabilitating people in a closed regime and deprived of some of their freedoms; through different processes, for example. Psychological assistance; Identification of intrinsic aspects of the inmates, that make them, are suitable to perform a certain task within the society; Beginning or culmination of the educational process, at the primary and secondary level. This last process, established as a study variable in the present research, with the purpose of identifying, if such a variable contributes in the process of rehabilitation of inmates, in the prison of women of Cusco in the period 2016. Thus, directed towards the objective formulated, the levels of performance and rehabilitation in which they are currently in that prison were identified, followed by the use of hypothesis tests, with the purpose of identifying the relation of yield towards the rehabilitation; through a descriptive correlational-causal cross-sectional, established design. From the analysis of the extracted data it was possible to conclude that the rehabilitation is not significantly affected by the academic performance of the internal students within the prison. It is evident that in this case the rehabilitation does not depend on the variable 'academic performance.'
\end{abstract}

\title{
KEYWORDS
}

Academic performance, rehabilitation, prison treatment boarders, establishment prison. 


\section{Introducción}

Uno de los problemas sociales relevantes y polémicos —que actualmente se evidencia en varios centros de reclusión penitenciarios situados dentro de los países de América Latina y el Caribe - es, si dentro de estos centros penitenciarios existen procesos encaminados a la rehabilitación de los confinados, que conduzcan a la inserción asertiva de los exinternos penitenciarios dentro de la sociedad. Muchos de estos centros penitenciarios adolecen de procesos coherentes y serios encaminados a la rehabilitación de los reclusos, puesto que la mayoría de estos centros presentan numerosos problemas como: sobrepoblación, impunidad, castigo como ideología, déficit en el sistema de justicia, capacitación y selección del personal penitenciario (Rangel, 2013); (Oliveira de Barros Leal, 2004), (Carranza, 2012). Estos problemas no son ajenos en la mayoría de los centros penitenciarios del Perú.

Sin embargo, dentro de las normas y reglamentos existen procedimientos encaminados hacia la rehabilitación de los internos para su reinserción en la sociedad puestos en práctica con sus limitaciones en diferentes centros penitenciarios del Perú. Uno de estos procedimientos, frecuentemente ejecutado en los diferentes centros de reclusión dentro del territorio nacional, está relacionado con la educación. Es el caso del Establecimiento Penitenciario de Mujeres del Cusco. Así, de acuerdo con el Código de Ejecución Penal, se debe fomentar y promover la educación del interno en todos los penales con la finalidad de conseguir su formación profesional o capacitación adecuada acorde a sus aptitudes, intereses y vocación.

La dirección de los establecimientos, a través de la administración penitenciaria, tiene la obligación de promover la educación artística, formación moral; así como la práctica de disciplinas deportivas que van a coadyuvar en la rehabilitación de los internos. Debemos reconocer que la educación penitenciaria es parte importante de la rehabilitación del interno, la que funciona como dispositivo de control que imparte conocimientos, normas, hábitos y valores a los estudiantes, induciendo a que el individuo se conduzca de acuerdo a las reglas — a lo correcto y qué no lo es-, que seleccione la opción más racional, tome decisiones acertadas y evalúe las consecuencias para asumirlas de forma responsable, que son signos de que hay alta probabilidad de rehabilitarse y, consecuentemente, está apto para reinsertarse a la sociedad, puesto que a través de la educación ha adquirido elementos necesarios para la reinserción social, la que es registrada en diferentes documentos manejados por los 
profesionales de psicología, asistencia social y otros, que tengan que ver con la rehabilitación del interno; ello en cumplimiento de la normatividad vigente de la entidad que los alberga, vale decir, el Instituto Nacional Penitenciario (INPE).

La educación penitenciaria (que responde a la normatividad vigente del Código de Ejecución Penal en convenio con el Ministerio de Educación del Perú) que se desarrolla dentro de algunos penales y presenta una realidad particular, compleja y quizás es la menos estudiada en el campo de la pedagogía — por la particularidad del estudiante y el difícil contexto de funcionamiento-; es medida verificando sus niveles alcanzados en las dimensiones procedimentales, conceptuales y actitudinales, a través del rendimiento académico.

Supuestamente, la educación en los penales contiene actividades encaminadas a contribuir en la rehabilitación del interno. La rehabilitación social de las personas reclusas consiste, en esencia, en suministrar herramientas para que progresivamente el interno se reeduque y se adapte permitiéndole alcanzar niveles adecuados en diferentes aspectos como físico, mental, social y espiritual; desarrollar habilidades en el sector productivo para su reinserción coherente y productiva en la sociedad. (Welch Reyes, 2014).

De acuerdo con los diferentes argumentos presentados, podríamos formularnos la siguiente pregunta: ¿existe una relación directa entre el rendimiento académico penitenciario y la rehabilitación de las internas estudiantes del Establecimiento Penitenciario de Mujeres del Cusco?, supuestamente podríamos afirmar que: el rendimiento académico penitenciario y la rehabilitación de las estudiantes internas del Establecimiento Penitenciario de Mujeres de Cusco, se relacionan directa y positivamente, lo que quiere decir — para un aumento paulatino en el nivel de aprendizaje - que existe un aumento progresivo en el nivel de rehabilitación. Tal aserto tendría que ser aceptado o negado a través del análisis de la información extraída del objeto de estudio. Esto nos sugiere realizar un análisis sobre el nivel de relación causal que existe entre el rendimiento académico y la rehabilitación asociadas a las reclusas estudiantes del Establecimiento Penitenciario de Mujeres del Cusco, así como la detección del grado ordinal en la que se encuentran actualmente las variables de estudio, de manera independiente. 


\section{Materiales y métodos}

Puesto que el presente trabajo de investigación está dirigido a proporcionar conocimiento basado en la información extraída de una realidad tal como se presenta para, posteriormente, ser analizada, contrastada y concluida; es posible sugerir que el presente trabajo de investigación se encuentra ubicado dentro del conjunto de trabajos de investigación que pertenecen al tipo de investigación descriptivo básico.

\section{Población y muestra}

De acuerdo al objetivo que persigue el presente trabajo de investigación, el objeto de estudio asociado a las variables en estudio está constituido por el total de reclusas que gozan del beneficio de los procesos que ofrece el área de educación del Establecimiento Penitenciario de Mujeres del Cusco, en las modalidades de Educación Básica Alternativa (EBA) y Educación Técnico Productivo (ETPRO); con la participación de 40 estudiantes internas.

\section{Técnicas e instrumentos}

La recuperación de información asociada al objeto de estudio, que corresponde a las variables de estudio — Rendimiento académico y Rehabilitación—, fue realizada siguiendo un proceso que involucra técnicas de análisis de documentos ya que los valores cuantitativos $\mathrm{y}$ cualitativos que corresponden a tales variables son atribuidos $\mathrm{y}$ registrados longitudinalmente durante periodos establecidos en documentos oficiales proporcionados por el Ministerio de Educación y el Establecimiento Penitenciario de Mujeres del Cusco; tal información fue recuperada y ordenada, de acuerdo con el propósito del trabajo de investigación, para luego ser registrada en instrumentos denominados 'registros de análisis de documentos.'

\section{Diseño de la investigación}

La información requerida por este trabajo de investigación, fue registrada en documentos pertinentes periódicamente durante el año 2016, por las áreas correspondientes dentro del penal de mujeres. Haciendo uso de estos documentos fue analizada y extraída la información, la cual quedó registrada en el instrumento denominado 'Registro de análisis de documentos'; en un solo instante de tiempo. Por otro lado, como el objetivo del presente trabajo es aceptar 
o negar la modificación de la rehabilitación por causa del rendimiento, es posible establecer un diseño de investigación descriptivo correlacional causal, de corte transversal, de acuerdo al esquema presentado en el grafico N. ${ }^{\circ} 1$

\section{Gráfico $\mathbf{N}^{\circ} 1$. Esquema del diseño de investigación}

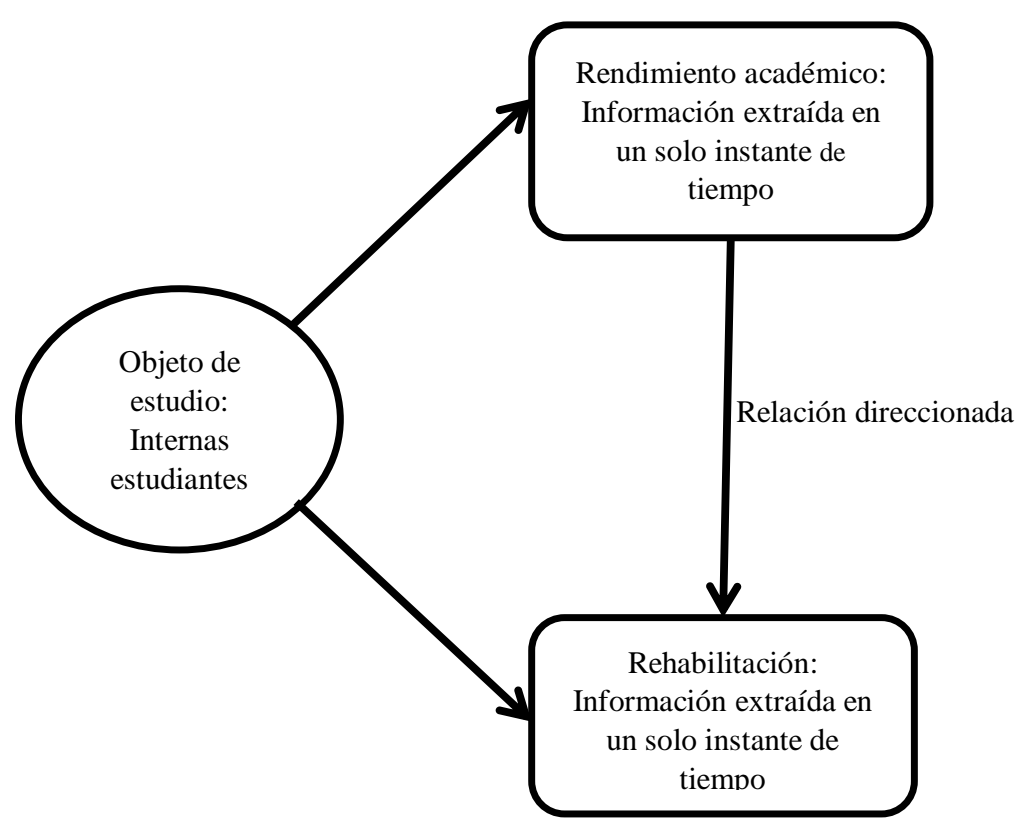

\section{Métodos de análisis de datos}

De acuerdo a los objetivos del presente trabajo de investigación, se hace necesario realizar un análisis descriptivo con la información proporcionada por el objeto de estudio; para lo cual se utilizaron algunas herramientas constituyentes de la estadística descriptiva, como por ejemplo: las frecuencias presentadas a través de tablas, gráficos descriptivos de barras y el cálculo de la media y moda, como elementos del conjunto de medidas de tendencia central. Con la finalidad de evidenciar la relación direccionada de variables (Rendimiento académico, Rehabilitación), se analizó la dependencia de variables mediante la prueba de hipótesis de Tau-c de Kendall, con un nivel de significancia del 0,05.

El proceso cuantitativo asociado a los métodos estadísticos fue realizado usando el software Excel Microsoft 210.0 y SPSS 21.0. 


\section{Resultados}

El cuadro $\mathrm{N}^{\circ}$, presenta la información referente a la variable de estudio: Rendimiento académico, categorizado en una escala de medición ordinal.

\section{Cuadro $N^{\circ} 1$ : Resultados categorizados correspondiente a la variable 'Rendimiento académico'}

\begin{tabular}{lcccc}
\multicolumn{1}{c}{ Variable } & Puntuación & Categoría & Frecuencia & \% \\
\hline \multirow{2}{*}{$\begin{array}{l}\text { Rendimiento } \\
\text { académico }\end{array}$} & De 12 & Nivel bajo & 4 & 10 \\
\cline { 2 - 5 } $\begin{array}{l}\text { De 13 a 14 } \\
\text { penitenciario }\end{array}$ & Nivel regular & 20 & 50 \\
\cline { 2 - 5 } & De 15 a 16 17 a 20 & Nivel alto & 16 & 40 \\
\cline { 2 - 5 } & & Nivel muy alto & 0 & 0 \\
\hline
\end{tabular}

El gráfico de barras correspondiente al cuadro 1 , se muestra en el gráfico $\mathrm{N} .^{\circ} 2$, donde se puede verificar que el $50 \%$ de las estudiantes internas obtuvieron una puntuación de 13 o 14, alcanzando un rendimiento regular, por otro lado, un $40 \%$ de las estudiantes internas obtuvieron una puntuación de 15 o 16, logrando un nivel alto. 


\section{Gráfico $\mathbf{N}^{\circ}$ 2: Resultados para la variable 'Rendimiento académico'}

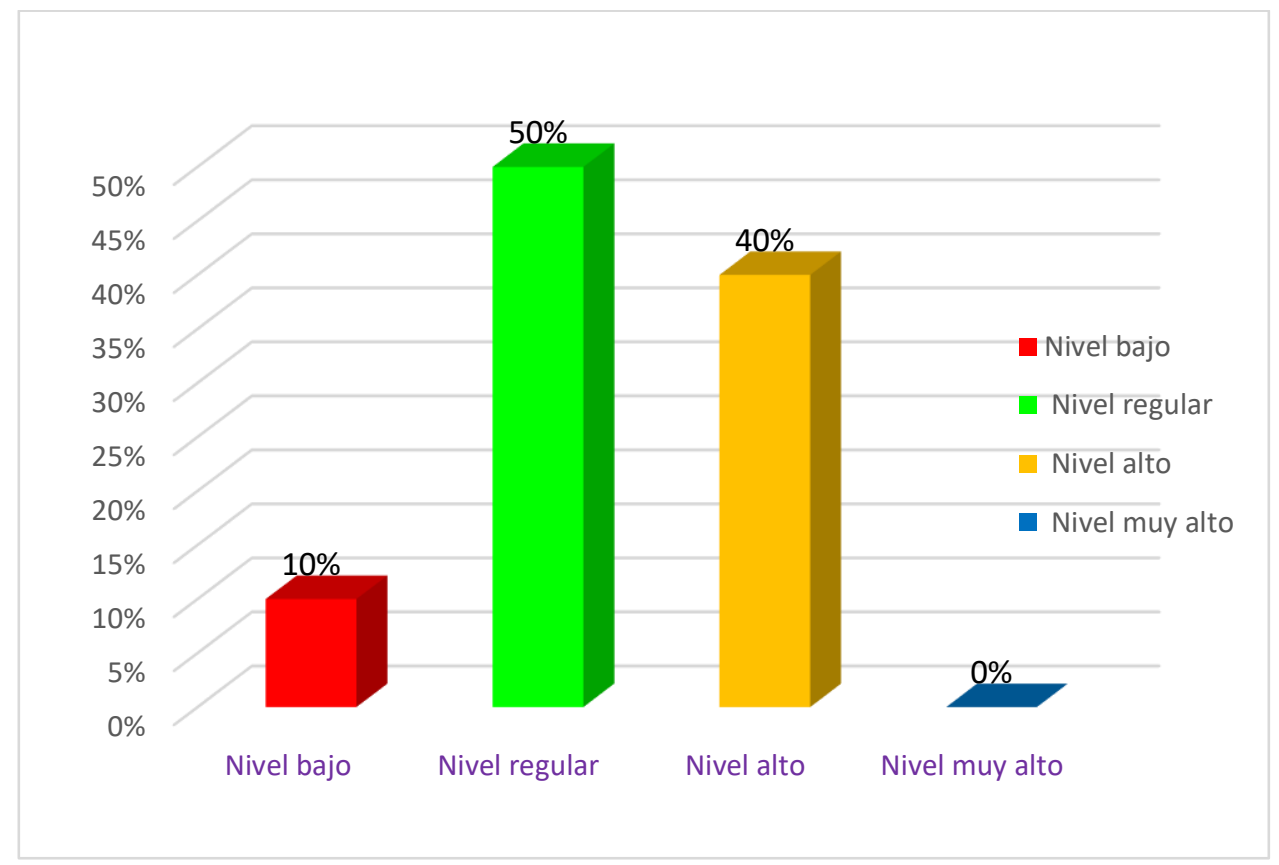

Los resultados consolidados que corresponden a la variable de estudio 'Rehabilitación', a través de resultados de sub variables asociadas (dimensiones), son presentados en el cuadro $\mathrm{N}^{\circ}$ 2, categorizado de acuerdo a una escala de medición ordinal.

Los resultados atribuidos a las dimensiones: Servicio social, Servicio psicológico y Seguridad; surgen como respuesta de las internas a todos los procesos que se desarrollan en el penal, asociados a cada una de estas sub variables.

Considerando a la variable 'Rehabilitación' como una conclusión de estas tres dimensiones, se podría afirmar que la rehabilitación en las internas del penal de mujeres de Cusco, se encuentra en un nivel medio con un $20 \%$, seguido de un nivel alto en un $80 \%$, como se observa en el grafico N. ${ }^{\circ} 3$.

El análisis individual, por cada dimensión se presenta en el gráfico $\mathrm{N} .^{\circ} 4$, donde se muestra que en un $27,5 \%, 12,5 \%$ y el 22,5\% se encuentran en nivel medio, y el $70 \%, 87,5 \%$, $77,5 \%$, en un nivel alto para cada dimensión, respectivamente. 


\section{Cuadro $N^{\circ}$ 2: Resultados categorizados correspondiente a la variable}

\section{'Rehabilitación'}

\begin{tabular}{|c|c|c|c|c|}
\hline Variable & Dimensiones & Categorías & Frecuencia & $\%$ \\
\hline \multirow{3}{*}{ REHABILITACIÓN } & SERVICIO SOCIAL & $\begin{array}{c}\text { Bajo } \\
\text { Medio } \\
\text { Alto } \\
\text { Total }\end{array}$ & $\begin{array}{c}1 \\
11 \\
28 \\
40\end{array}$ & $\begin{array}{c}2,5 \\
27,5 \\
70.0 \\
100.0\end{array}$ \\
\hline & $\begin{array}{c}\text { SERVICIO } \\
\text { PSICOLÓGICO }\end{array}$ & $\begin{array}{c}\text { Bajo } \\
\text { Medio } \\
\text { Alto } \\
\text { Total }\end{array}$ & $\begin{array}{c}0 \\
5 \\
35 \\
40\end{array}$ & $\begin{array}{c}0.0 \\
12,5 \\
87,5 \\
100.0\end{array}$ \\
\hline & $\begin{array}{l}\text { NORMAS DE } \\
\text { SEGURIDAD }\end{array}$ & $\begin{array}{c}\text { Bajo } \\
\text { Medio } \\
\text { Alto } \\
\text { Total }\end{array}$ & $\begin{array}{c}0 \\
9 \\
31 \\
40\end{array}$ & $\begin{array}{c}0.0 \\
22,5 \\
77,5 \\
100.0\end{array}$ \\
\hline \multicolumn{2}{|c|}{ TOTAL VARIABLE } & $\begin{array}{c}\text { Bajo } \\
\text { Medio } \\
\text { Alto } \\
\text { Total }\end{array}$ & $\begin{array}{c}0 \\
8 \\
32 \\
40\end{array}$ & $\begin{array}{c}0.0 \\
20.0 \\
80.0 \\
100.0\end{array}$ \\
\hline
\end{tabular}

Grafico $N^{\circ}$ 3: Resultados para la variable 'Rehabilitación'

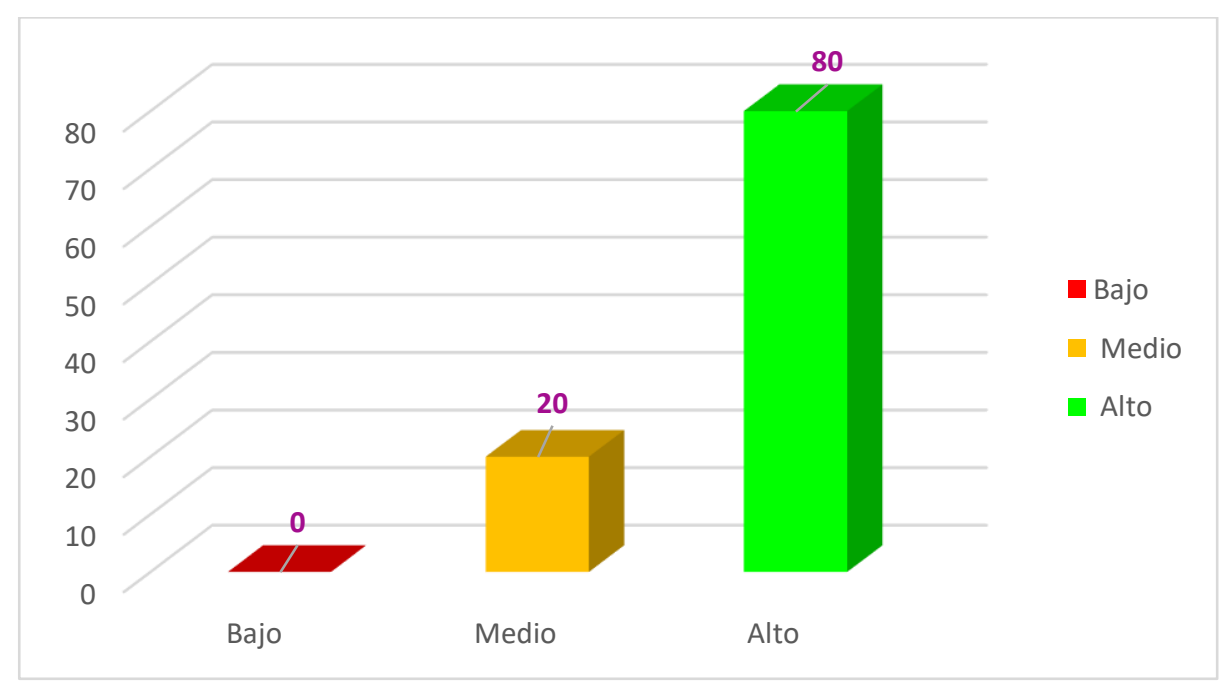




\section{Gráfico N 4: Resultados para la variable 'Rehabilitación'}

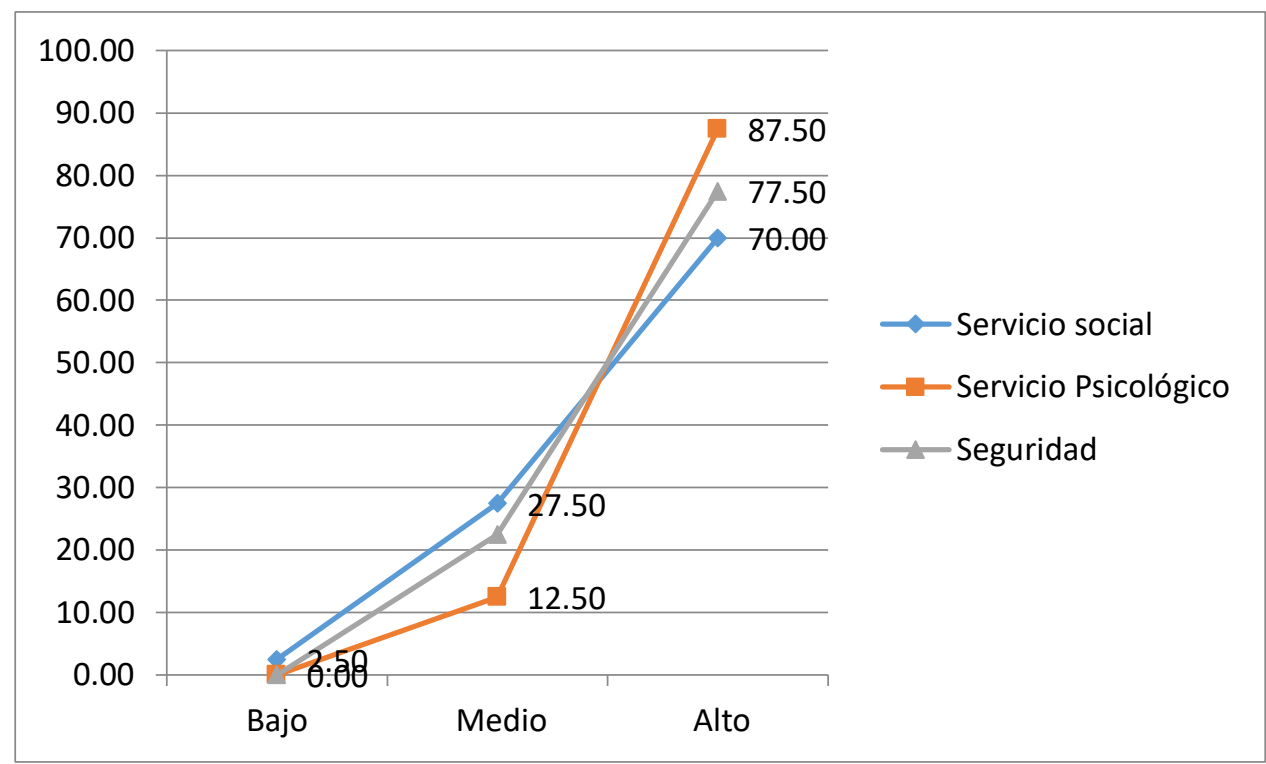

Los resultados orientados a determinar la relación entre las variables de estudio se presentan en el cuadro $\mathrm{N} .{ }^{\circ} 3$, donde se muestran los valores de probabilidad (p) correspondientes a la aceptación o negación de la hipótesis nula estadística, esta última asociada a la negación de una posible relación entre las variables de estudio. En ese entender, se puede afirmar, que según los datos mostrados, las variables 'Rendimiento académico' y 'Rehabilitación', no tienen ninguna relación significativa; puesto que el valor de probabilidad obtenido resulta en un valor $(0,88)$, superior al valor de significancia 0,05 , lo que sugiere aceptar la hipótesis nula.

Para la variable 'Rendimiento académico' y la dimensión 'Servicio social', se observa que sí existe una relación significativa $(0,042<0,05)$, lo cual sugiere afirmar, que existen algunos factores involucrados en el 'Rendimiento académico' que inciden en el 'Servicio social.' 


\section{Cuadro $\mathbf{N}^{\circ}$ 3: Resultados del p- valor para la prueba de Tau-c de Kendall}

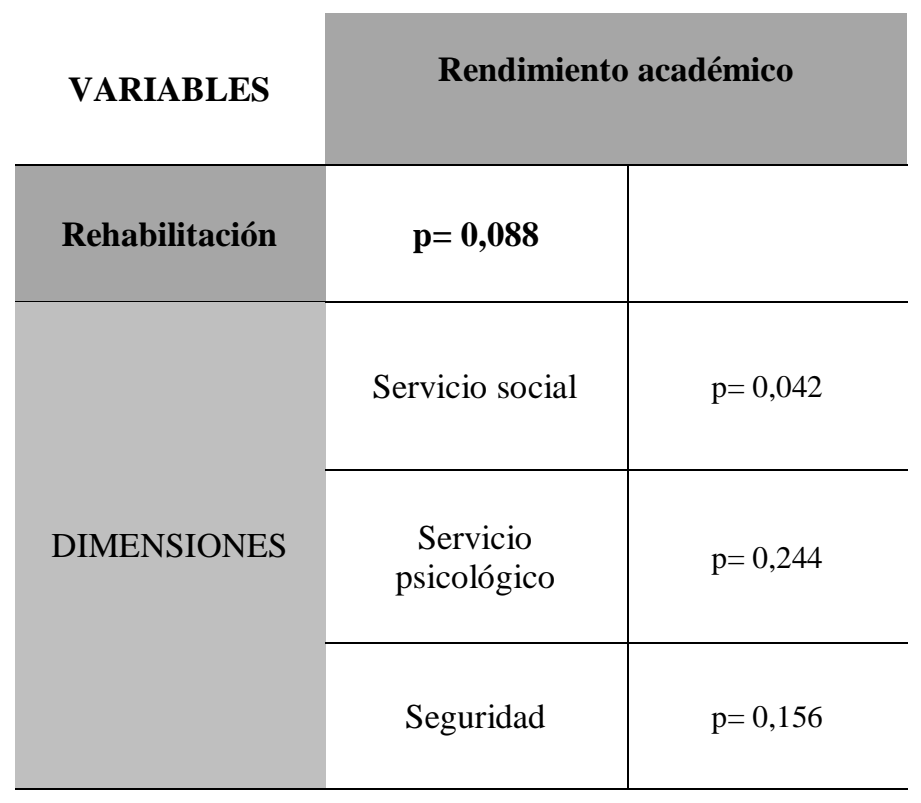

\section{Discusión}

Si fuera posible identificar todos los factores involucrados en las diferentes variables asociadas a las múltiples actividades formales y no formales que se realizan dentro de los penales (Ruano M. R. \& Mendoza H. Y., 1979); (Soto B.G \& Luengo A. D. , 2015); existiría la posibilidad adicional de poder reforzar tales factores, con la finalidad de elevar los niveles de rehabilitación. Cabe recalcar que a pesar de que algunos de estos factores, por definición teórica estén encaminados en ayudar a la rehabilitación de internos recluidos dentro de los penales, dependerán del contexto espacio temporal y de las personas encargadas en realizar actividades que involucran estos factores, con el fin de obtener resultados esperados en cuanto se refiere a la rehabilitación. Así, teóricamente es coherente suponer, que actividades relacionadas con la educación estructurada y sistematizada contribuirá en algunos aspectos relacionados al proceso de rehabilitación (Cisneros, 2015). En este caso, se evidenció que el 'Rendimiento académico', como uno de los factores de medida de los niveles alcanzados dentro del servicio de educación, no se relaciona significativamente con la rehabilitación. El nivel alto, obtenido en el 'Rendimiento académico' en las internas del penal de Cusco, no 
contribuye significativamente a la rehabilitación, a pesar que este tiene una relación significativa con la dimensión 'Servicio social', constituyente de la variable 'Rehabilitación'. En este caso, se podría suponer que el rendimiento académico incidirá significativamente en otros aspectos que no están relacionados con la rehabilitación.

\section{Conclusiones}

De acuerdo a la información existente en el periodo correspondiente al año 2016, extraída del objeto de estudio «reclusas del penal de mujeres de Cusco»; analizada, estructurada y contrastada; se establece que el rendimiento académico dentro del penal de mujeres del Cusco, se encontraba en un nivel entre regular y alto.

Por otro lado, la rehabilitación, que está constituida por diferentes elementos como: servicio social, servicio psicológico y seguridad, en el periodo 2016, se encontraba en un nivel alto, resultado este que no es, precisamente, por la incidencia del rendimiento académico, puesto que se evidenció la independencia significativa entre las dos variables: rendimiento académico y rehabilitación.

Como el objetivo principal del Establecimiento Penitenciario de Mujeres del Cusco, es obtener niveles de rehabilitación elevados, conseguidos en el menor tiempo posible, se hace necesario sugerir la evaluación de los elementos que constituyen los procesos educacionales relacionados al centro penitenciario, para su mejor direccionamiento hacia la rehabilitación.

\section{Conflictos de interés}

Los autores declaran no tener conflictos de interés en la publicación de este artículo. 


\section{Referencias Bibliográficas}

Carranza, E. (2012). Situación penitenciaria en América Latina y el Caribe ¿Qué hacer? Anuario de Derechos Humanos(0 (8)), 31 - 66. doi:10.5354/0718-2279.2012.20551

Cisneros, J. L. (2015). La presbicia de la educación en prisión, $\mathrm{n}^{\circ} 1$,. Revista de Historia de las Prisiones, $1\left(\right.$ N. $^{\circ}$ 1), pp. 34-52. doi:ISSN: 2451-6473

Oliveira de Barros Leal, C. (2004). La pena de prisión en América Latina: Los privados de libertad y sus derechos humanos. Revista do instituto brasileiro de direitos humanos, 14, 105-112. Obtenido de http://milas.x10host.com/ojs/index.php/ibdh/article/view/265

Rangel, H. (1 de jan./mar de 2013). Educación contra corriente en las cárceles latinoamericanas: La enseñanza vs el castigo. (F. d.-U.-P. Alegre, Ed.) Educação \& Realidade, 38(n. 1), p. 15-32. Obtenido de http://www.ufrgs.br/edu_realidade/

Ruano M. R. \& Mendoza H. Y. (1979). Rehabilitación del delincuente, un estudio sobre actitudes y opiniones de familiares de internos. ILANUD . Costa Rica: Naciones Unidas. Obtenido de http://www.justicia.gob.ec/wp-content/uploads/2015/08/REHABILITACION-DELDELINCUENTE-ILANUD.pdf

Soto B.G. \& Luengo A. D. (Marzo de 2015). Factores asociados al alta terapéutica del tratamiento de drogas en población penal chilena. . Boletín . Observatorio chileno de drogas (N. $\left.{ }^{\circ} 18\right)$. doi: 0719 - 2770

Welch Reyes, Y. R. (2014). La reeducación y reinserción social del recluso en Centroamérica. Tesis de grado, Universidad Rafael Landívar, Quetzaltenango. Recuperado el 2017, de http://biblio3.url.edu.gt/Tesario/2014/07/01/Welch-Yeysson.pdf 